\title{
Emergence of current branches in a series array of negative differential resistance circuit elements
}

\author{
Huidong $\mathrm{Xu}$ and Stephen W. Teitsworth ${ }^{\mathrm{a})}$ \\ Department of Physics, Duke University, P.O. Box 90305, Durham, North Carolina 27708-0305, USA
}

(Received 1 June 2010; accepted 12 July 2010; published online 18 August 2010)

\begin{abstract}
We study a series array of nonlinear electrical circuit elements that possess negative differential resistance and find that heterogeneity in the element properties leads to the presence of multiple branches in current-voltage curves and a nonuniform distribution of voltages across the elements. An inhomogeneity parameter $r_{\max }$ is introduced to characterize the extent to which the individual element voltages deviate from one another, and it is found to be strongly dependent on the rate of change of applied voltage. Analytical expressions are derived for the dependence of $r_{\max }$ on voltage ramping rate in the limit of fast ramping and are confirmed by direct numerical simulation. (C) 2010 American Institute of Physics. [doi:10.1063/1.3475988]
\end{abstract}

\section{INTRODUCTION}

Negative differential resistance (NDR) - in which an increasing applied voltage causes a reduced electrical current flow-occurs in a number of electronic transport systems, for example, tunnel diodes, ${ }^{1}$ semiconductor double barrier quantum well structures, ${ }^{2}$ and molecular bridges. ${ }^{3,4}$ When several such elements are placed in a series array, forming a complex system, the NDR of individual elements often leads to striking self-organization effects associated with a spatially nonuniform distribution of electric field, despite the fact that the elements are almost identical. An important example of this behavior is provided by semiconductor superlattices consisting of a series array of quantum wells (e.g., composed of GaAs) separated by potential barriers (e.g., composed of $\mathrm{AlAs})$. In the case of doped superlattices with relatively wide barrier layers (i.e., weakly-coupled superlattices), one may observe multiple current branches in current-voltage $(I-V)$ characteristics with the number of branches approximately equal to the number of periods of the superlattice. ${ }^{5,6}$ These branches are associated with a static, nonuniform electric field configuration in which some periods of the structure are in a low-field domain and the others in a high-field domain. ${ }^{7,8}$ Current branching and/or nonuniform electric field distributions have been reported for other spatiallyperiodic systems such as quantum cascade laser structures, multiple-quantum well infrared detectors, ${ }^{10} \mathrm{Si}$ nanocrystal structures, ${ }^{11}$ and two-dimensional transport in laterally patterned GaAs quantum wells. ${ }^{12}$ It is remarkable that, despite the microscopic differences between these systems, the observed behavior is similar. This naturally raises two questions: what are the minimal ingredients needed to observe current branch formation? What is the role of heterogeneity in the individual element properties on this behavior?

In this paper, we address these questions by introducing a nonlinear circuit model consisting of a voltage-biased series array of $N$ ideal NDR elements, each connected in parallel to a capacitance as shown in Fig. 1(a). We find that

${ }^{a)}$ Electronic mail: teitso@phy.duke.edu. heterogeneity in the element properties leads to the formation of multiple branches in $I-V$ curves and a nonuniform distribution of voltages across individual elements.

\section{NONLINEAR CIRCUIT MODEL AND FIELD INHOMOGENEITY PARAMETER}

The model is defined as follows. Each element of the series array is assumed to have an intrinsic $I-V$ curve of the form $I_{i}\left(v_{i}\right)$ with a typical example shown in Fig. 1(b) ${ }^{13}$ The total electrical current is

$$
I=I_{i}\left(v_{i}\right)+C_{i} \dot{v}_{i},
$$

for $i=1,2, \ldots, N$, where $v_{i}$ denotes the voltage across the $i$ th element and $C_{i}$ is the parallel capacitance associated with the $i$ th element. The total applied voltage to the array is $V$ $=\sum_{j=1}^{N} v_{j}$. Dividing both sides of Eq. (1) by $C_{i}$ and summing over $i$ allows to express the total current as

$$
I=C_{t o t} \dot{V}+C_{t o t} \sum_{i=1}^{N} I_{i} / C_{i},
$$

where $C_{\text {tot }}^{-1} \equiv \sum_{i=1}^{N} C_{i}^{-1}$ denotes the inverse total equivalent series capacitance. Relabeling the summation in Eq. (2) as over $j$ and substituting the result back into Eq. (1), the circuit model equations can be written in the following form:
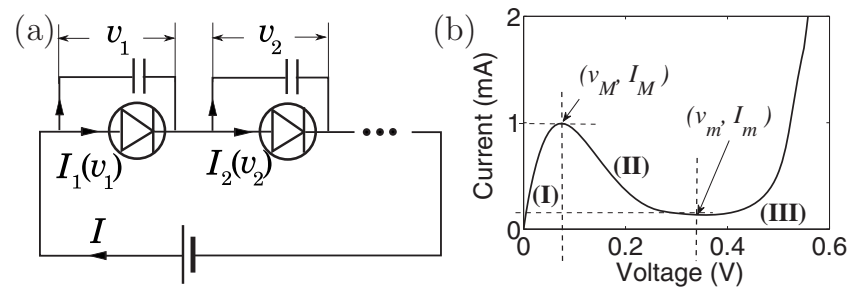

FIG. 1. (a) Nonlinear element array model. NDR elements are connected in series with parallel capacitance associated to each element. (b) The $I-V$ curve for a typical element. Region (II) is the NDR region. 


$$
\dot{v}_{i}=\frac{C_{\text {tot }}}{C_{i}} \dot{V}+\sum_{j=1}^{N} K_{i j}\left[I_{j}\left(v_{j}\right)-I_{i}\left(v_{i}\right)\right]
$$

where $K_{i j}=C_{t o t} / C_{i} C_{j}$. Equation (3) has the form of an $\mathrm{N}$-dimensional dynamical system subject to a constraint on the total voltage. The constraint is built into the structure of the model, as seen by summing Eq. (3) over all $i$ and using the symmetry property $K_{i j}=K_{j i}$. The structure of Eq. (3) is equivalent to a limiting case of a standard rate equation model for superlattices, when the doping level in each quantum well is very large. ${ }^{7,14,15}$

In this paper, we focus on the case of linear ramping (with ramp time $T$ ) in the total applied voltage $V$, so that $V$ increases linearly between values 0 and $N V_{\max }$ and $\dot{V}$ $=N\left(V_{\max } / T\right) \equiv N \alpha$. The first term in Eq. (3) describes the effect of changing total applied voltage, while the second term describes a global coupling between individual circuit elements. When the ramping rate is large, the effect of the coupling term is expected to be small. On the other hand, when the ramping rate is small, the coupling term plays a decisive role in the observed behavior. The coupling term displays either positive or negative feedback depending on the state of each element. Considering pairs of elements, they attract one another when both are on a stable branch of the $I-V$ curve [i.e., regions I and III in Fig. 1(b)], and repel if they are both on the unstable part (region II). If one element is in a stable region and the other is in the unstable region, the elements may either attract or repel depending on their relative current values.

If the coupling term in Eq. (3) is neglected, one finds an uncoupled state in which the element voltages increase independently. Starting from the initial condition $v_{i}^{(0)}(0)=0$ for all $i$, typical for experimental measurements, ${ }^{7,9,12}$ the voltage of each element is given by $v_{i}^{(0)}=V \times C_{t o t} / C_{i}$. Since the $C_{i}$ are assumed to have a small dispersion, the uncoupled state is associated with a field profile that is nearly uniform. (Here, we use the term field to refer to the spatial distribution of element voltages.) When the coupling term is nonzero, the system deviates from the uncoupled state and the field profile becomes nonuniform. This behavior is usefully characterized by introducing a field inhomogeneity parameter $r_{\max }$ defined by

$$
r_{\max }=\max \{r(t): 0<t<T\},
$$

where

$$
r(t)=\frac{1}{N} \sum_{j=1}^{N}\left(\frac{v_{j}-v_{j}^{(0)}}{v_{j}^{(0)}}\right)^{2} .
$$

The quantity $r(t)$ expresses the time-dependent level of field nonuniformity in the system for ramp time $T$, while $r_{\max }$ gives the maximal degree of nonuniformity during the entire ramping process and associates a single value to the entire process. For the uncoupled state, $r_{\max }$ tends to zero; however, when the effect of the coupling term is large, $r_{\max }$ assumes a value of order 1 .

If the total voltage is held constant, the system always relaxes to a state in which the currents in the different elements are identical. This corresponds to a stable fixed point
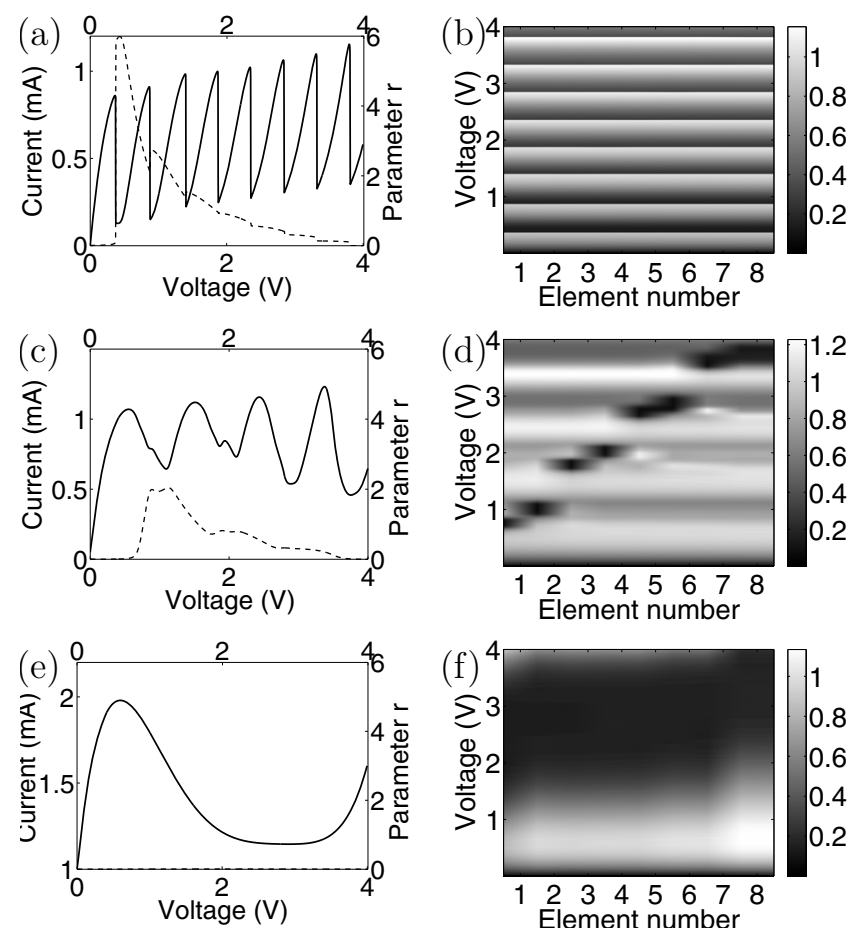

FIG. 2. $I-V$ curves and current level contour plots for different ramp times, all with $N=8, C=200 \mathrm{nF}$, and $\sigma_{I}=0.1$. (a) Fully coupled case: $I-V$ curve with ramp time $T=500 \mathrm{~ms}$. Dashed curve shows the parameter $r(t)$. (b) Current contour plot for $T=500 \mathrm{~ms}$ (gray scale in units milliampere). (c) $I-V$ curve for the partially coupled case with $T=2 \mathrm{~ms}$. (d) Current contour plot for $T=2 \mathrm{~ms}$. (e) $I-V$ curve for the uncoupled case with $T=0.1 \mathrm{~ms}$. (f) Current contour plot for $T=0.1 \mathrm{~ms}$.

of the system with $\dot{V}=0$. If the average applied voltage, $V / N$, falls in the NDR region of the single element $I-V$ curve, there are multiple fixed points corresponding to distinct arrangements of individual element voltages. For a completely homogeneous system, such that all elements are identical, i.e., $C_{i}=C$ and $I_{i}(v)=I_{0}(v)$ for all $i$, these fixed points are degenerate corresponding to the same overall device current. ${ }^{16}$ When the homogeneous system is subjected to a ramped voltage, starting from the initial state $v_{i}(t=0)=0$ for all $i$, the element voltages remain identical throughout the ramp process, i.e., $v_{i}(t)=V(t) / N$, and the overall $I-V$ curve has a similar shape to that of a single element. ${ }^{17}$

\section{RESULTS AND ANALYSIS}

When heterogeneity is introduced into the system, current branches emerge in the limit of slow ramping as shown in the $I-V$ curve of Fig. 2(a). The variance in the individual element $I-V$ characteristics is expressed as $I_{i}\left(v_{i}\right)=I_{0}\left(v_{i}\right)(1$ $+\epsilon_{i}$ ), where the $\epsilon_{i}$ 's are independent and identically distributed random numbers, distributed normally with mean zero and standard deviation $\sigma_{I}$, i.e., $\sigma_{I}^{2} \equiv \frac{1}{N} \sum_{i=1}^{N} \epsilon_{i}^{2}$. ${ }^{18}$ We have also explored the effect of capacitance variation and variation due to electrical noise in individual elements, and find that the qualitative behavior is the same as that observed when the only variance is in the element $I-V$ curves. ${ }^{19}$

When the ramp time $T$ is large, the system exhibits welldefined current branches in the static $I-V$ curves, shown in Fig. 2(a) for $N=8$. As the total voltage ramps higher, the elements pass very rapidly from region I to III one by one, 
and the current exhibits an abrupt step down for each such passage. ${ }^{20}$ The parameter $r_{\max }$ takes a value of order 1 , indicative of a nonuniform distribution of element voltages. Figure 2(b) shows the corresponding contour plot in which the current level is plotted in gray scale vs element number $i$ and total voltage $V$. The clear horizontal bands indicate that, for all parts of the ramping process, the individual device currents are identical to one another.

As the ramp time decreases, the element current levels become somewhat different from one another, see Fig. 2(c). In this case, more than one element can jump to region III at the same time. The field distribution deviates less from the uniform state, the current branches are rounded and smaller in number and amplitude, and the abrupt jumps between current branches disappear. A similar rounding of experimental current branches versus ramping rate has been reported in weakly-coupled GaAs/AlAs semiconductor superlattices. ${ }^{21}$ The $r_{\max }$ value also decreases from its large $T$ value. Figure 2(d) shows the corresponding current contour plot in which the horizontal bands are still evident, but interrupted by localized dark areas that correspond to the passage of individual or pairs of elements through region II.

For fast ramping, the element currents are essentially independent of one another, and the element voltages pass through the NDR region simultaneously [Fig. 2(e)]. The $r_{\max }$ parameter approaches zero, implying that the system behavior is very close to the uncoupled state described previously. The $I-V$ curve of the full array follows closely that of an individual element, $I_{0}(v)$. The contour plot, Fig. 2(f), shows smooth behavior and horizontal features are absent. We have also considered ramping from different initial states ${ }^{19}$ as well as more elaborate circuit array models-e.g., including small series inductance and parallel capacitance with each nonlinear element ${ }^{22}$ - and find a qualitatively similar behavior as described above.

Plotting the value of $r_{\max }$ versus ramp time $T$ for several different $N$ values in Fig. 3(a) indicates that the transition from uncoupled to fully coupled behavior is a smooth transition with an onset that is independent of system size $N$. For large ramp time, $r_{\max }$ approaches an asymptotic value that is $N$ dependent. As $N \rightarrow \infty, r_{\max } \longrightarrow 2.85$, a value that depends only on the shape of the single element function $I_{0}(v) .{ }^{19}$ For the parameters used here (i.e., $C=200 \mathrm{nF}$ and $\sigma_{I}=0.1$ ), the transition from uncoupled to coupled behavior occurs as the ramp time increases from $\sim 100 \mu$ s to $\sim 10 \mathrm{~ms}$, a timescale range that is significantly greater than the zero-bias characteristic $R C$ time constant associated with an individual element, i.e., $\tau=C d I_{0} /\left.d v\right|_{v=0} \simeq 2 \mu$ s.

To better understand this behavior, we investigate the effect of perturbations about the uncoupled state of the form

$$
v_{i}=v_{i}^{(0)}+\delta v_{i} \equiv v+\delta v_{i}
$$

where $v_{i}^{(0)}(t)=\alpha t$ denotes the uncoupled state solution. Substituting this form into the dynamical model, Eq. (3), one finds the following system of differential equations valid to first order in the $\epsilon_{i}$ 's:
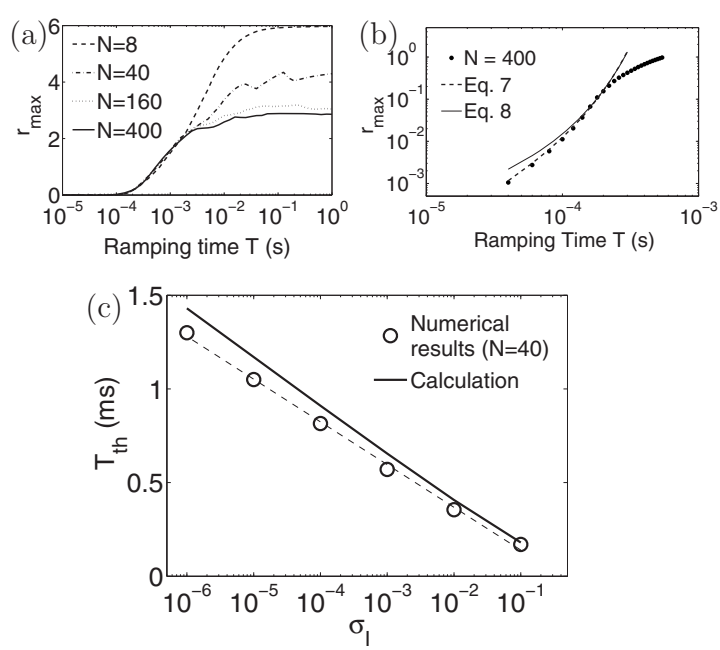

FIG. 3. (a) The parameter $r_{\max }$ vs ramp time $T$ for indicated values of $N$. Each curve is an average of ten different statistical configurations of the $I_{i}\left(v_{i}\right)$ curves with $C=200 \mathrm{nF}$ and $\sigma_{I}=0.1$. (b) Closed circles give $r_{\max }$ vs $T$ near the characteristic value for $N=400$. The solid curve is a first-order calculation using Eqs. (4) and (7), while the dashed curve is based on Eq. (8). (c) $T_{t h}$ vs $\sigma_{I}$, comparing analytical prediction with simulation for $N$ $=40$.

$$
\begin{aligned}
\delta \dot{v}_{i} & =\frac{1}{N C}\left\{I_{0}(\alpha t) \sum_{j=1}^{N}\left(\epsilon_{j}-\epsilon_{i}\right)+I_{0}^{\prime}(\alpha t) \sum_{j=1}^{N}\left(\delta v_{j}-\delta v_{i}\right)\right\} \\
& =-\frac{I_{0}(\alpha t)}{C} \epsilon_{i}-\frac{I_{0}^{\prime}(\alpha t)}{c} \delta v_{i},
\end{aligned}
$$

where $I_{0}^{\prime} \equiv d I_{0} / d v$. Equation (6) can be solved explicitly to yield the following expression for $\delta v_{i}$ :

$$
\delta v_{i}=-\frac{\epsilon_{i}}{C \alpha} \mathrm{e}^{-I_{0}(v) / C \alpha} \int_{0}^{v} I_{0}\left(v^{\prime}\right) \mathrm{e}^{I_{0}\left(v^{\prime}\right) / C \alpha} d v^{\prime},
$$

with the initial conditions $\delta v_{i}(0)=0$ for all $i$.

For large values of ramping rate $\alpha$, the two exponentials in Eq. (7) are approximated by unity, and it immediately follows that

$$
\begin{aligned}
\delta v_{i}(t) & =-\frac{\epsilon_{i}}{c} \int_{0}^{t} I_{0}\left(\alpha t^{\prime}\right) d t^{\prime}=-\frac{\epsilon_{i}}{c \alpha} \int_{0}^{v} I_{0}\left(v^{\prime}\right) d v^{\prime} \\
& \equiv-\frac{\epsilon_{i}}{c \alpha} P(v) .
\end{aligned}
$$

Substituting this result into the definition of $r_{\max }$, see Eqs. (4) and (5), allows us to write

$$
r_{\max }=\frac{\sigma_{I}^{2} T^{2}}{c^{2} V_{\max }^{2}} \max [P(v) / v]^{2},
$$

which demonstrates that $r_{\max }$ is proportional to $T^{2}$ with a coefficient of proportionality that is independent of $N$; this is confirmed in Fig. 3(a).

For somewhat smaller $\alpha$ values, and provided the local maximum of the element $I-V$ curve is sufficiently sharp, Eq. (7) can be evaluated using a saddle point method to write $\delta v_{i} \approx \frac{\epsilon_{i} I_{M} \sqrt{\pi}}{\sqrt{\beta c \alpha}} \mathrm{e}^{\frac{I_{M}-I_{0}(v)}{C \alpha}}$, where $\beta=-I^{\prime \prime}(v) /\left.2\right|_{v_{M}}>0$. Inserting this result back into the definition of $r(t)$, see Eq. (5), gives 


$$
r(v)=\frac{\pi \sigma_{I}^{2} I_{M}^{2} T}{\beta C V_{\max } v^{2}} \mathrm{e}^{2\left[I_{M}-I_{0}(v)\right] / C \alpha} .
$$

In the preceding equation, we note that the exponential term takes a maximum value if we set $v=v_{m}$, so that $I_{M}-I_{0}(v)$ $=I_{M}-I_{m} \equiv \Delta I$, where $v_{m}$ and $I_{m}$ are the voltage and current coordinates of the local minimum in the element $I-V$ characteristic, see Fig. 1(b). This allows us to write the maximum value of $r$ over the entire ramp process as

$$
r_{\max }=\frac{\pi \sigma_{I}^{2} I_{M}^{2} T}{\beta C V_{\max } v_{m}^{2}} \mathrm{e}^{2 \Delta I / C \alpha} .
$$

Figure 3(b) plots the values of $r_{\max }$ calculated from both Eqs. (7) and (8) and compares them with the numerical results for $N=400$, demonstrating that there is a range of ramp times (around $10^{-4}$ seconds) for which the asymptotic expression, Eq. (8) follows closely the transition from uncoupled to coupled behavior. In order to develop an analytic criterion for the onset of coupled behavior-and associated field nonuniformity, we define a characteristic value of ramp time $T_{t h}$ that sits in this range. Denoting the corresponding characteristic value of $r_{\max }$ by $r_{t h}$, we have $\ln r_{t h}=2 \ln \sigma_{I}$ $+\ln \left(\left(\pi I_{M}^{2} / C \beta v_{m}^{2}\right)\right)-\ln \alpha+(2 \Delta I / \alpha C)$. Solving for $T_{t h}$, we can write

$$
T_{t h}=-\frac{C V_{\max }}{\Delta I} \ln \sigma_{I}+K,
$$

where $\quad K=\left(C V_{\max } / \Delta I\right)\left((1 / 2) \ln \left(r_{t h} \beta C V_{\max } v_{m}^{2} / \pi I_{M}^{2}\right)\right.$ $\left.-(1 / 2) \ln T_{t h}\right)$ is slowly varying. Equation (9) shows explicitly the dependences of $T_{t h}$ on element heterogeneity (i.e., $\sigma_{I}$ ) as well as the single element NDR behavior. Interestingly, the prefactor of the $\ln \sigma_{I}$ term, calculated to be $C V_{\max } / \Delta I$ $\simeq 1.18 \times 10^{-4} \mathrm{~s}$, has the form of an $R C$ time with an effective resistance that depends only on the total current drop across the NDR region, $\Delta I$. In particular, this time scale is not sensitive to the shape details of the $I_{0}(v)$ curve in the NDR region. Figure 3(c) plots $T_{t h}$ versus the variance level (with the specific choice $r_{t h}=0.1$ ) and shows good agreement with numerical data for $N=40$.

\section{SUMMARY}

We have introduced a simple model that reveals how NDR and element heterogeneity are key sources for the observation of nonuniform field distributions and multiple current branches in the electrical conduction properties of a series array of nonlinear circuit elements. Specifically, we have shown how the system approaches a state in which the element current levels are fully coupled as the element variance level and voltage ramp rate are varied. The numerical and analytical results obtained from this model provide insight for understanding similar observed behaviors that are found for a range of more complex electronic systems, for example, semiconductor superlattices and quantum cascade laser structures. $^{7-9}$ In practical devices, it is often desirable to have an electric field distribution that is as spatially uniform as possible. The model introduced here points out the relevance of two factors for achieving such field distributions. One of these factors is the effective level of element heterogeneity in a periodic structure, which, in a device such as a superlattice, is determined in part by the fabricated sample quality and the electrical noise level. The second factor suggests the possible use of a time-dependent voltage bias that possesses a ramping rate through the NDR region of the device that is large enough to maintain a nearly uniform field distribution.

\section{ACKNOWLEDGMENTS}

We gratefully acknowledge helpful conversations with Luis Bonilla, Gleb Finkelstein, Eckehard Schöll, and Adrienne Stiff-Roberts. This work was supported by the National Science Foundation through Grant No. DMR-0804232.

${ }^{1}$ L. Esaki, Phys. Rev. 109, 603 (1958).

${ }^{2}$ V. J. Goldman, D. C. Tsui, and J. E. Cunningham, Phys. Rev. Lett. 58, 1256 (1987)

${ }^{3}$ J. Chen, M. A. Reed, A. M. Rawlett, and J. M. Tour, Science 286, 1550 (1999).

${ }^{4}$ R. Liu, S.-H. Ke, H. U. Baranger, and W. Yang, J. Am. Chem. Soc. 128, 6274 (2006).

${ }^{5}$ K. K. Choi, B. F. Levine, N. Jarosik, J. Walker, and R. Malik, Phys. Rev. B 38, 12362 (1988).

${ }^{6}$ H. T. Grahn, R. J. Haug, W. Müller, and K. Ploog, Phys. Rev. Lett. 67, 1618 (1991)

${ }^{7}$ L. L. Bonilla and H. T. Grahn, Rep. Prog. Phys. 68, 577 (2005).

${ }^{8}$ E. Schöll, Nonlinear Spatio-Temporal Dynamics and Chaos in Semiconductors (Cambridge University Press, Cambridge, 2001).

${ }^{9}$ S. L. Lu, L. Schrottke, S. W. Teitsworth, R. Hey, and H. T. Grahn, Phys. Rev. B 73, 033311 (2006)

${ }^{10}$ H. Schneider, C. Schönbein, R. Rehm, M. Walther, and P. Koidl, Appl. Phys. Lett. 88, 051114 (2006).

${ }^{11}$ J. Chen, J. J. Lu, W. Pan, K. Zhang, X. Y. Chen, and W. Z. Shen, Nanotechnology 18, 015203 (2007).

${ }^{12}$ W. Pan, S. K. Luo, J. L. Reno, J. A. Simmons, D. Li, and S. K. J. Brueck, Appl. Phys. Lett. 92, 052104 (2008).

${ }^{13}$ For specificity, we use for the function $I_{0}(v)$ a fifth-order polynomial fit to the measured $I-V$ curve of a tunnel diode (model 1N3712).

${ }^{14} \mathrm{H}$. Xu and S. Teitsworth, Phys. Rev. B 76, 235302 (2007).

${ }^{15}$ H. Xu, Ph.D. thesis, Duke University, 2010.

${ }^{16}$ J. C. Neu, private communication (23 July 2008).

${ }^{17} \mathrm{By}$ contrast, the use of nonuniform initial conditions has an equivalent effect as introducing element heterogeneity to the system.

${ }^{18}$ We use a Gaussian distribution on $[-1,1]$ and have found that the observed behavior is not sensitive to the detailed form of distribution.

${ }^{19}$ Huidong $\mathrm{Xu}$ and Stephen Teitsworth, (unpublished).

${ }^{20}$ For very small $N$, one may observe smooth current branches that do not end in discontinuous current jumps, even in the static limit $\alpha \rightarrow 0$. Using Eq. (3) with the first term set to zero, one may estimate the minimum number of elements $N_{\min }^{(1)}$ necessary for the first branch to end in an abrupt current jump, namely, $N_{\min }^{(1)}=1+g_{1} /\left|g_{2}\right|$, where $\left|g_{2}\right|$ denotes the magnitude of the maximal negative differential conductance in region II of figure and $g_{1}$ denotes the positive differential conductance in region I, evaluated at the same current level as $\left|g_{2}\right|$. Similar conditions may be derived for each of the separate current branches.

${ }^{21}$ M. Rogozia, S. Teitsworth, H. Grahn, and K. Ploog, Phys. Rev. B 65, 205303 (2002)

${ }^{22}$ Kevin Brown and Stephen Teitsworth, (unpublished). 